\title{
Problèmes de Génie civil concernant certains types d'installations pneumatiques de transfert d'énergie
}

\author{
par J. Raud \\ Département “Matériels", S.E.P.T.E.N. \\ E.D.F., Paris
}

Les systèmes d'accumulation d'énergie sous forme pneumatique peuvent se classer en deux catégories principales:

- les systèmes purement pneumatiques, sans compensation hydraulique;

- les systèmes avec compensation hydraulique, ou hydropneumatique.

Parmi les promoteurs de ces derniers systèmes, nous citerons particulièrement: des établissements suédois (la firme Stal-Laval, d'importants bureaux d'étude de génie civil suédois), et $\mathrm{M}$. Djordjevic en Yougoslavie.

Nous ne parlerons, ici, que des procédés utilisant des cavernes souterraines. Ceux qui emploient des structures de roches poreuses se rattachent au premier genre car au cours d'un cycle d'une ou quelques heures l'eau n'a pas le temps de migrer dans les couches et ils fonctionnent donc comme systèmes sans compensation hydraulique. Un exemple de cette méthode de stockage en formation sableuse a été étudié en France.

\section{Premières comparaisons entre les deux systèmes avec réservoirs en cavernes}

Les systèmes sans compensation hydraulique sont certainement bien plus simples, au point de vue du génie civil, que ceux qui emploient la méthode hydropneumatique. Mais ils nécessitent des réservoirs beaucoup plus grands.

L'utilisation de la compensation hydraulique permet d'avoir une pression sensiblement constante. La valeur de cette pression est imposée par la profondeur du réservoir souterrain au-dessous du réservoir de surface puisqu'elle est créée par la charge d'eau correspondant à cette profondeur.

Avec les systèmes sans compensation hydraulique, les pressions varient au cours du temps mais on a plus de liberté pour leur choix en fonction de la profondeur, ce qui peut être un avantage important.

Faisons une remarque concernant les installations avec compensation hydraulique:

L'énergie théoriquement productible à partir de l'air stocké dans un réservoir d'installation hydropneumatique peut être considérée comme étant la somme de deux termes :

- le premier terme représente l'énergie mécanique que l'on produirait dans une installation sans compensation hydraulique avec un réservoir de même volume et de même pression maximum, en dégonflant complètement ce réservoir de la pression maximum à la pression atmosphérique; l'air est supposé utilisé sans laminage intermédiaire dans une turbine à gaz fictive pouvant fonctionner sous pression variable;

- le deuxième terme représente l'énergie mécanique que produirait une turbine hydraulique utilisant les mêmes réservoirs supérieur et inférieur, ce dernier étant maintenu à la pression atmosphérique.

\section{Conditions requises des réservoirs, en relation avec la valeur de la pression}

Notons tout de suite que, pour des raisons économiques, la plus grande partie des réservoirs ne peut pas être revêtue et que la roche doit donc rester à nu. Les réservoirs doivent satisfaire à deux conditions essentielles : étanchéité et stabilité des parois. Voyons quelques relations de ces conditions avec la pression:

En ce qui concerne l'étanchéité, il est souhaitable (sauf en terrain complètement imperméable) que la pression dans le réservoir ne soit pas supérieure à la pression interstitielle dans le terrain. Il peut être nécessaire de créer une mise en charge interstitielle artificielle au moyen d'un réseau de galeries, ce qui est assez coûteux. D'après une étude suédoise $(*)$, un tel réseau pourra être efficace et utile si la perméabilité de la roche est comprise entre $10^{-5}$ et $10^{-8} \mathrm{~m} / \mathrm{s}$.

(*) D. Noren, L. O. Emmeling, S. E. Paulson, Storing energy underground A swedish development. V.B.B. Stockholm (Engineering - 4 septembre 1970). 
Au point de vue de la résistance à la rupture, la pression ne devra pas dépasser une certaine fraction (les 3/4 par exemple) de la contrainte qui règne dans le terrain à ce niveau.

Dans le cas de terrains non complètement imperméables, ce sera en général la condition d'étanchéité qui fournira la limite supérieure de la pression admissible. Pour répondre à cette condition, la compensation hydraulique implique d'ailleurs que la pression interstitielle croisse avec la profondeur, ce qui ne sera le cas que sur certains sites, et l'on voit pourquoi les méthodes purement pneumatiques sont plus souples à ce point de vue.

Compte tenu des conditions requises, il serait bien fortuit de rencontrer une cavité naturelle ou artificielle toute faite pouvant convenir.

On peut penser à une galerie ou un réseau de galeries creusées dans des terrains d'excellente qualité.

\section{Description sommaire d'une installation hydropneumatique}

Prenons comme exemple le cas d'une installation hydropneumatique dont le réservoir souterrain serait situé à $240 \mathrm{~m}$ sous le réservoir de surface. La pression dans le réservoir souterrain sera donc voisine de 24 bars relatifs (25 bars absolus).

On suppose ici que l'on utilise une turbine à gaz d'un type commercialisé dont voici quelques caractéristiques dans les conditions d'utilisation normales :

- température des gaz entrant dans la tur.

bine. . ................. $870^{\circ} \mathrm{C}$

- puissance de l'alternateur dans le type commercialisé. ..................

$57,35 \mathrm{MW}$

— puissance d'un alternateur entraîné par

la turbine seule.............. 160 MW

— rapport de compression. ........... 9,3

- débit d'air aspiré.......... environ $345 \mathrm{~kg} / \mathrm{s}$

Sous 25 bars absolus et à $25^{\circ} \mathrm{C}$, le débit volume sera d'environ $11,8 \mathrm{~m}^{3} / \mathrm{s}$. C'est aussi le débit d'eau nécessaire.

Nous admettons un temps d'utilisation journalier de $4 \mathrm{~h} / \mathrm{j}$ (14 $400 \mathrm{~s}$ ) en production, et de $8 \mathrm{~h} / \mathrm{j}$ en pompage. Le volume utile du réservoir souterrain devra donc être de : $170000 \mathrm{~m}^{3}$ soit, compte tenu de fuites diverses: environ $180000 \mathrm{~m}^{3}$. Enfin, des gardes doivent être ménagées, en partie haute pour la circulation d'air et en partie basse pour celle de l'eau. Compte tenu de ces gardes, nous prévoyons un volume réel de 200000 à $210000 \mathrm{~m}^{3}$.

Avec la turbine ci-dessus, nous comptons donc qu'il faut, pour un réservoir de $240 \mathrm{~m}$ de profondeur: $0,266 \mathrm{~m}^{3}$ utile par kWh.

Compte tenu des caractéristiques des turbines données dans la communication précédente, on trouve pour la même profondeur de $240 \mathrm{~m}$, des valeurs des volumes utiles suivants :

$0,266 \mathrm{~m}^{3}$ utiles/ $\mathrm{kWh}$ avec la turbine de 9,3 bars ci-dessus,

$0,163 \mathrm{~m}^{3}$ utiles $/ \mathrm{kWh}$ avec une turbine de 9,8 bars, à injection d'eau,

$0,236 \mathrm{~m}^{3}$ utiles $/ \mathrm{kWh}$ avec une turbine à $1000^{\circ} \mathrm{C}$ sans injection d'eau,

$0,210 \mathrm{~m}^{3}$ utiles/ $\mathrm{kWh}$ avec une turbine de 20 bars, à deux corps, sans réchauffe entre corps,
$0,142 \mathrm{~m}^{3}$ seulement, si l'on disposait d'une turbine de 13 bars, $1000^{\circ} \mathrm{C}$, à injection d'eau.

Pour transformer ces «volumes utiles》 en volumes réels de réservoirs, il faut les multiplier par un facteur de l'ordre de 1,2 tenant compte de pertes d'air diverses et des gardes.

Nous avons limité actuellement la profondeur du réservoir à une valeur de $250 \mathrm{~m}$, mais il est évident qu'une augmentation de cette profondeur diminuerait le volume du réservoir nécessaire.

Dans les figures 1,2 et 3 , nous avons considéré un réservoir constitué par un faisceau de galeries d'une dizaine de mètres de diamètre. Dans les zones voisines des extrémités, on a admis un revêtement mais celui-ci grève le coût de l'ensemble. Le diamètre de $10 \mathrm{~m}$ n'est donné qu'à titre d'exemple. Si le terrain le permet, il sera plus économique d'augmenter la section et éventuellement de prévoir une section plus haute que large.

Les alimentations en air et eau peuvent se faire de diverses façons. Dans plusieurs publications, ces alimentations sont prévues à partir d'une section unique qui est située au droit d'un nœud du réseau de galeries. Nous avons adopté ici une disposition dans laquelle cette alimentation se fait à partir d'extrémités différentes: une pour l'eau et une pour l'air.

Le puits est unique. Il sert à l'adduction de l'eau entre le réservoir de surface et le réservoir souterrain. Sa dimension est telle que la vitesse de l'eau y soit faible en pompage: il est, en effet, souhaitable d'avoir une vitesse faible au cours de la remontée de l'eau.

Le puits communique avec l'ouvrage d'entrée d'eau du réservoir par un siphon, dont le but est de favoriser la stabilité hydraulique du système. Une petite cavité en fond du puits sert de réserve de sédimentation.

Le puits contient aussi le tube de circulation de l'air. L'air débouche, par une galerie, dans une chambre située à la partie haute du réservoir souterrain, avant de pouvoir pénétrer dans le réservoir lui-même.

Cette disposition permet de constituer le réservoir souterrain par des galeries de section sensiblement constantes.

\section{Problèmes posés par les aménagements à compensation hydraulique, concernant les installations souterraines}

De tels aménagements nous paraissent poser certains problèmes que nous allons examiner sommairement: les uns intéressent les terrains, les autres les mouvements des fluides dans les installations souterraines.

\section{Problèmes concernant les terrains.}

Ce sont, pensons-nous, parmi les principaux. Nous avons déjà signalé qu'il n'était pas possible d'envisager un revêtement complet, mais tout au plus celui de certaines zones particulières telles que les chambres d'air et d'eau aux extrémités du réservoir et certains tronçons du réseau de galeries.

Or, les réservoirs sont remplis alternativement d'air et d'eau avec un rythme quotidien. Leurs parois doivent pouvoir résister à ces actions. Les terrains doivent donc présenter d'excellentes qualités mécaniques et avoir une grande résistance chimique vis-à-vis de l'eau et de l'air. Des terrains granitiques profonds sont sans doute envisageables, s'ils ne contiennent pas de minéraux altérables et à la condition qu'ils soient sains et non fissurés. Il peut en être de même 


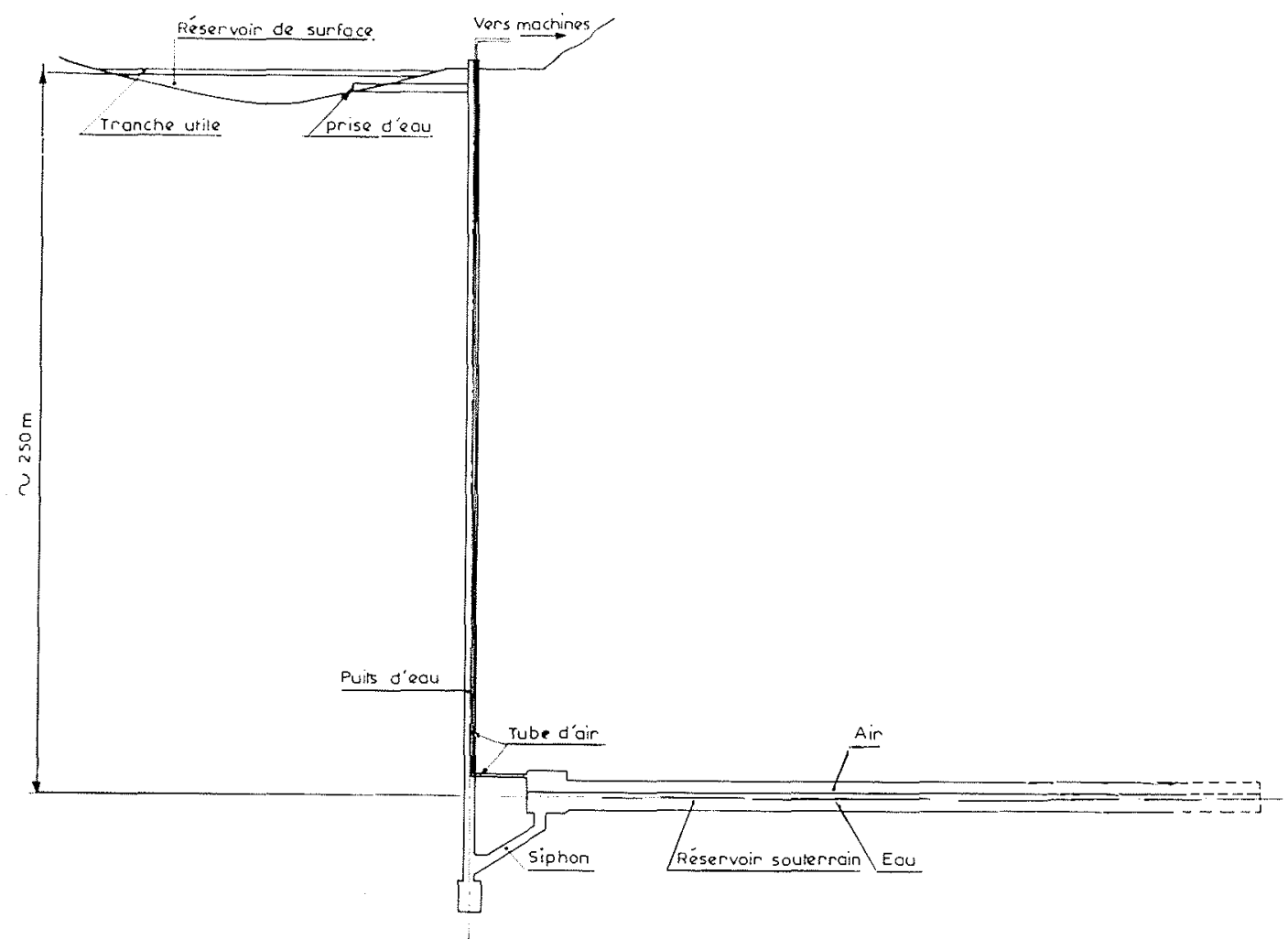

1/Schéma de principe d'installation avec compensation hydraulique.

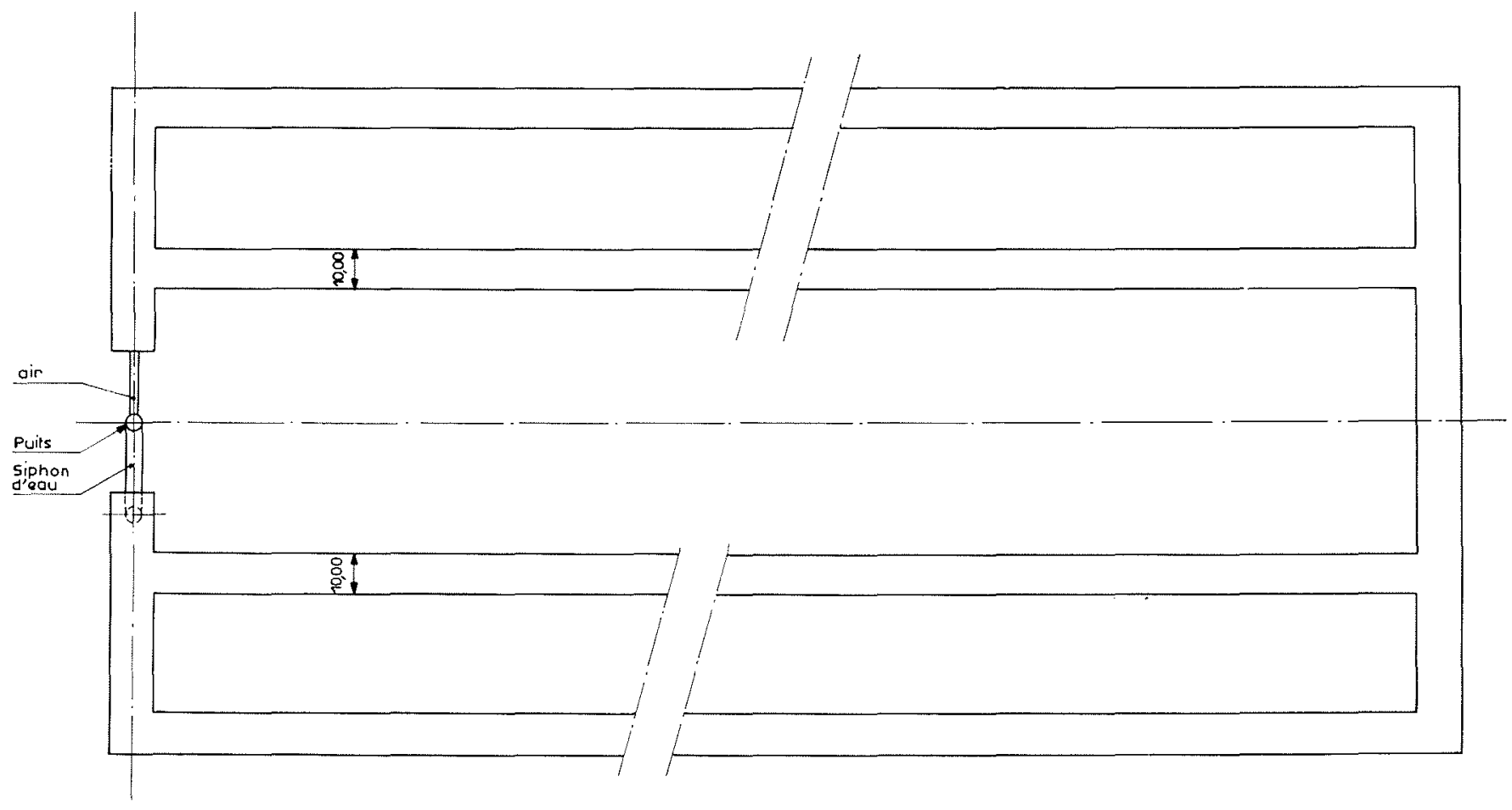

2/ Plan schématique de réservoir (exemple). 


\section{J. RAUD}

de certains calcaires, s'ils sont suffisamment résistants et s'ils se trouvent dans une situation hydrologique convenable.

Sur le plan purement mécanique de l'équilibre des parois, le fait que la pression soit à peu près constante est une circonstance favorable. Cela réduit, en effet, la valeur des «sous-pressions»d'air et d'eau, qui peuvent prendre naissance dans les pores de la roche à proximité des parois nues et derrière les revêtements. Un clouage de la paroi ou un ancrage des revêtements au terrain seront favorables à la stabilité. L'emploi de revêtements en béton projeté sera souvent intéressant.

Bien entendu, les précautions à prendre tant sur les parois brutes que sur les parois revêtues seraient à préciser au moyen d'essais préalables.

Aux effets chimiques et mécaniques s'ajoutent ceux des phénomènes thermiques, qui peuvent provoquer des effets défavorables à la tenue des parois. Ils proviennent de variations de température dans les réservoirs, dues à deux causes possibles: la température de l'air injecté, et les variations de pression.

Or, l'air sortant des compresseurs est à une température telle qu'il ne peut évidemment pas être injecté directement dans le réservoir. Il faudra done prévoir des échangeurs qui ramènent sa température à une valeur voisine de celle de l'eau. Cela entraîne par ailleurs, un surcroît d'investissements et la nécessité de disposer de la capacité d'évacuation des calories.

D'autre part, la pression dans le réservoir souterrain n'est pas tout à fait constante, par suite des marnages et des pertes de charge diverses. Les variations de pression provoquent des variations de température de l'air, par compression ou détente. Mais si les premières ne dépassent pas quelques pour cent, les variations de température seront de quelques degrés seulement. Il pourrait n'en être pas de même dans le cas d'aménagements à faible profondeur, avec des variations de pressions relativement plus importantes.
Ecoulements d'air et d'eau dans les terrains.

Une forte pression interstitielle de l'eau dans les pores du terrain est favorable à l'étanchéité naturelle du réservoir. Est-ce suffisant? Les ingénieurs pétroliers constructeurs de réservoirs souterrains pour hydrocarbures gazeux ont là dessus une grande expérience.

Si la pression interstitielle est trop faible, un dôme d'air s'amorcera au-dessus du réservoir, et à partir d'un certain moment le phénomène peut devenir instable avec formation d'un cylindre d'écoulement d'air jusqu'à la surface du sol.

Dans certains cas, nous l'avons déjà signalé, on peut parfaire l'étanchéité au moyen d'un réseau de galeries de surpression interstitielle.

\section{Problèmes de mouvements de fluides dans les réservoirs et les canalisations}

Les écoulements des fluides dans les réservoirs et les canalisations sont assez complexes:

- lorsque le réservoir est plein d'eau, il doit néanmoins subsister une section de passage suffisante pour l'air afin que celui-ci ait une vitesse acceptable. Compte tenu de sa densité, il risque en effet de créer une agitation de la surface de l'eau qui entraînerait une érosion de la voûte. Une certaine «garde» est donc à prévoir en partie haute du réservoir;

- lorsque le réservoir est plein d'air, les mouvements d'eau doivent rester assez tranquilles dans le réseau de galeries et une garde est donc nécessaire aussi, en partie basse du réservoir.

Certains problèmes de stabilité sont liés aux variations de niveau d'eau dans le réservoir. Celles-ci peuvent avoir plu-

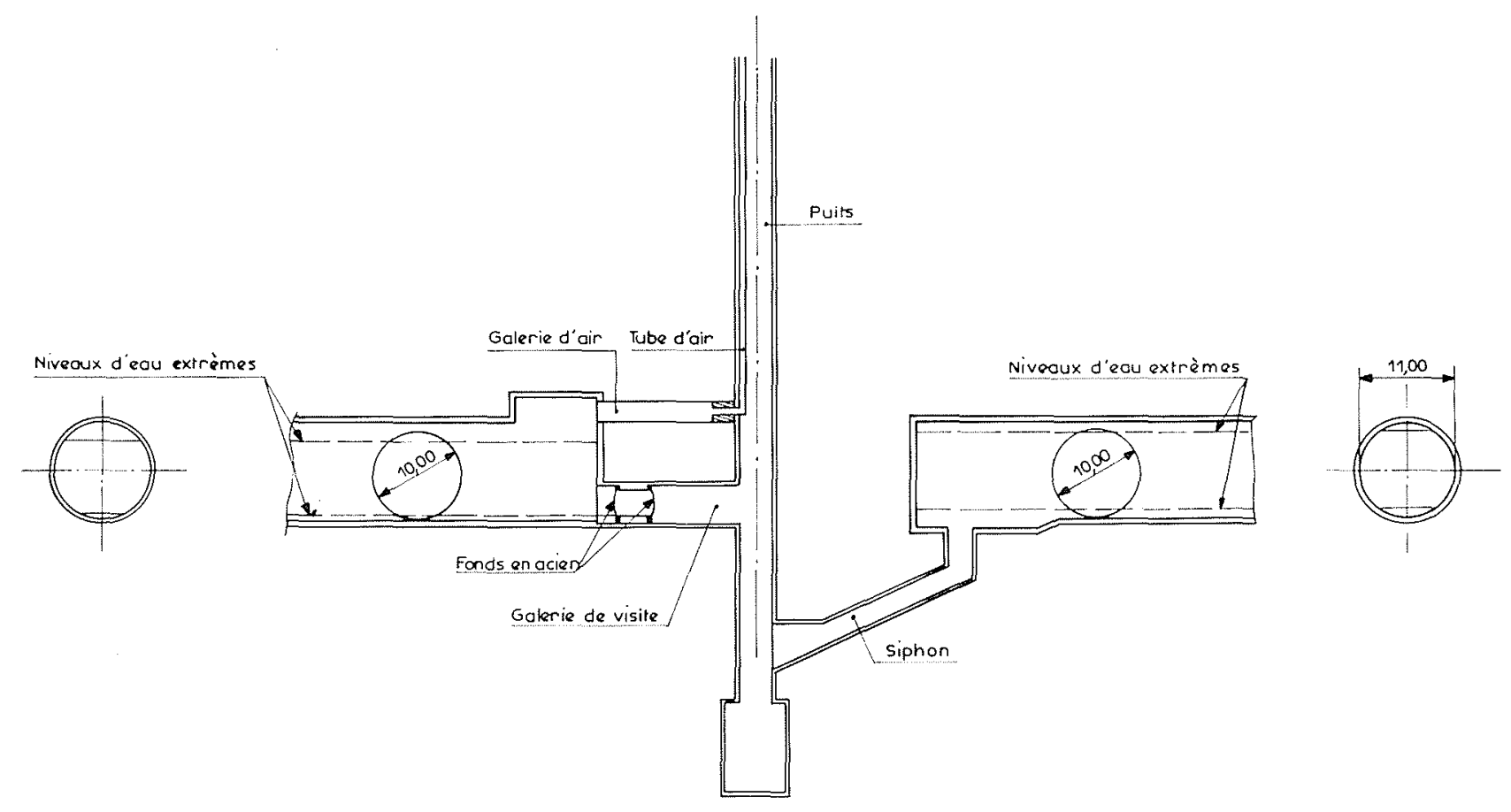

$3 /$ Schéma de départs d'air et d'eau. 
sieurs causes: oscillations de la surface libre, dégazage dans le puits qui diminue la densité fictive de l'eau; marnage du bassin supérieur dans certains cas. Pour éviter un reflux de l'air vers le puits d'eau, nous pensons qu'il est nécessaire de prévoir un siphon suffisamment profond à la base du puits.

Examinons maintenant la dissolution de l'air comprimé dans l'eau. La lenteur de ce phénomène maintiendra sans doute la quantité d'air dissous à une valeur assez éloignée de la saturation de l'eau. Néanmoins, il se peut que cette quantité soit assez forte pour qu'un dégazage de l'eau se produise dans le puits au cours de sa remontée. Un tel dégazage peut avoir des conséquences diverses:

- il ne doit pas être trop brutal;

- lorsqu'un dégazage prend naissance, il diminue la densité moyenne de l'eau du puits et donc la pression dans le réservoir souterrain: c'est une des raisons du risque d'instabilité, qui doit pouvoir être évité par un siphon;

- il entraîne quelques pertes d'air.

Il semble indiqué de prévoir une vitesse de remontée de l'eau faible, comme le recommandent certaines publications suédoises (*).

Notons enfin le problème de l'envasement, problème qui peut être important. Il faut éviter l'obstruction par les vases, des points bas de l'installation. La retenue de compensation

(") Cf. par exemple E. K. A. OLsson, $220 \mathrm{MW}$ Air storage power plant. A.S.M.E., Gas Turbine Conference, May 1970 devra donc être située sur une rivière aux eaux propres et la prise d'eau assez éloignée du fond.

Le siphon envisagé plus haut peut créer quelques difficultés à ce point de vue. Une chambre de sédimentation à la base du puits sert de dépôt aux sédiments inévitables; il est peut-être possible de prévoir un dispositif approprié qui permette de les extraire en permanence.

\section{Conclusion}

Nous avons indiqué dans cet exposé quelques problèmes de génie civil que nous paraissent poser certains types d'aménagements de stockage d'énergie par accumulation pneumatique. Ces problèmes concernent d'une part la tenue des terrains, d'autre part les mouvements des fluides utilisés. Ce sont les premiers qui nous paraissent les plus importants.

L'emploi de la compensation hydraulique permet d'emmagasiner, par mètre cube de réservoir, une quantité d'énergie bien plus grande que l'accumulation pneumatique pure. La constance de la pression est un facteur favorable sur plusieurs points. Toutefois, la présence des deux fluides air et eau aggrave certainement les conditions de tenue des parois des réservoirs et nécessite, à notre avis, d'être très exigeant vis-à-vis des aspects géologiques et topographiques des implantations de telles installations, si du moins on veut éviter de coûteux travaux confortatifs. 